\title{
backstory
}

\section{Mission Mars}

\section{Robin Fergason and colleagues sent a rover to Meridiani Planum to better understand the history of the red planet.}

\begin{abstract}
What are the objectives of the Mars Exploration Rover (MER) Mission that landed at Meridiani Planum?

The primary objective of this mission, which is still operating today, is to understand past environmental conditions at Meridiani Planum (a large plain near the equator of Mars), and to determine if it was once a favourable environment for life. Secondary goals include understanding the physical, chemical and mineralogical diversity of Meridiani Planum, and how that diversity corresponds to remote-sensing observations obtained from orbiting satellites and Earth-based instruments.
\end{abstract}

\section{Why was Meridiani Planum chosen as a landing site for this mission? \\ The Thermal Emission Spectrometer onboard the Mars Global Surveyor identified the mineral hematite from orbit. This mineral usually forms in water and has been observed in very few locations on Mars; of these, Meridiani Planum harbours the highest concentration and the largest areal extent, and so provides an excellent opportunity to understand whether Mars was once a favourable environment for life. Furthermore, the location is very desirable from a landing perspective. So, all in all, choosing to land at Meridiani Planum was an easy decision.}

\section{What sorts of data are being collected?}

As power is typically the limiting constraint on Mars, targets are carefully selected for observation. We collect images of the Meridiani Planum landscape using navigation and panoramic cameras, and obtain high-resolution images of very small regions using a microscopic imager. The mineralogy of rocks and surface layers,

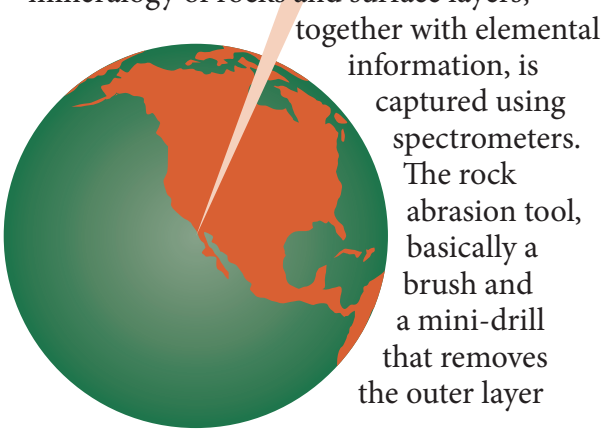

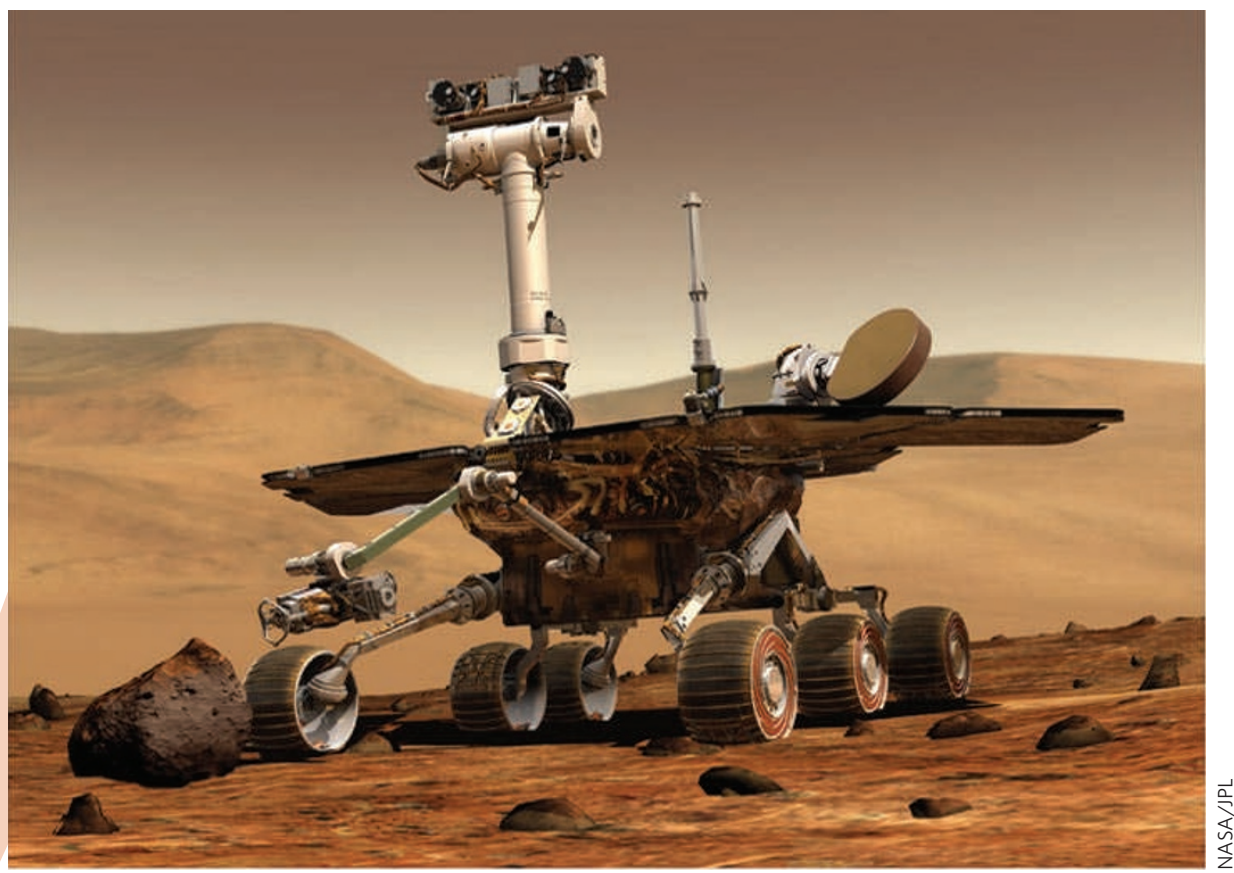

Roving round Mars. A computer-generated image of the Mars Exploration Rover.

of rocks, enables a clean surface to be analysed and provides information about the hardness of the rock surface.

\section{Any highlights?}

Reaching the edge of Victoria crater and getting our first glimpse of its scale and complexity, as well as having the opportunity to explore, was amazing. The rovers were only expected to travel a few hundred metres, yet Victoria crater sits about six kilometres from the landing site. At the beginning of the mission, we never dreamt we would travel so far.

\section{Have you learned anything new about} yourself or your team members?

We operated on Mars time during the primary mission. That means our day was approximately 40 minutes longer than a normal Earth day, hence our wake-up time, work start-time and so on shifted accordingly. Some people thrived on arriving at work at 3:30 am one week and $7: 15$ am the next, whereas others really hated it. I think people with families had the hardest time because they continually had to shift back and forth between both worlds. I thought Mars time was great! Imagine having an extra 40 minutes each evening.

\section{Has the team encountered any difficulties? \\ During the past five years we've} encountered many difficulties; Mars is an extreme environment. We've endured dust storms that reduced our solar power supply. We've been stuck in sand drifts and, at times, had more difficulty climbing up slopes than expected. And air-fall dust continues to accumulate on our solar panels. It's a testament to the persistence and creativity of the Mars Exploration Rover science and engineering teams that the scientific experiments and expeditions have been minimally compromised by these challenges. Oh, and the rover drivers can manoeuvre the vehicles into practically any position - they're incredible.

Robin Fergason of the US Geological Survey wrote this backstory to the work by Paul Niles and Joseph Michalski, published on page 215 of this issue. 